\title{
Identifying Factors Predicting Long-Term Opioid Use After Mastectomy
}

\author{
Sarah M. DeSnyder, MD \\ Department of Breast Surgical Oncology, The University of Texas MD Anderson Cancer Center, Houston, TX
}

In the 1990s, a surge in opioid prescriptions occurred in response to the declaration by the Joint Commission on Accreditation of Healthcare Organizations that pain is the "fifth vital sign." The resulting opioid epidemic has spurred efforts by health care providers, particularly surgeons, to decrease opioid prescribing while maintaining equivalent postoperative pain control.

Recommendations for breast surgical oncology procedures often are grouped with general surgery procedures and seem to assume one size fits all. For many breast surgeons, the question remains, "how do we determine the appropriate number of pills for each individual patient?"

In this study, Woeste et al. ${ }^{1}$ sought to identify risk factors for long-term opioid use by women undergoing mastectomy. A single-institution database was used to identify women from Kentucky who underwent mastectomy for either risk reduction or cancer treatment between January 2016 and December 2017. The study was limited to Kentucky residents because the Kentucky All-Schedule Prescription Electronic Reporting (KASPER) system was used to determine when the last opioid prescription was filled.

Consistent with prior reports in the literature, the authors defined prolonged opioid use (POU) as use for 90 days or longer after surgery. ${ }^{2}$ The authors found that patients using opioids in the preoperative setting, those with psychiatric illness, and those provided with a larger discharge prescription of opioids were more likely to have POU.

(C) Society of Surgical Oncology 2020

First Received: 20 December 2019;

Published Online: 5 February 2020

S. M. DeSnyder, MD

e-mail: sgainer@mdanderson.org
Notably, this study presumed that all prescriptions were filled and taken as prescribed. Given this assumption, opioid-naïve patients in this study were presumed to have an average opioid use duration of 113 days. However, prior studies have demonstrated that clinicians overestimate postoperative opioid needs. ${ }^{3,4}$ One study reviewed opioid use by breast surgical oncology patients at postoperative day 7 and demonstrated a prescribing excess of more than 220,000 oral morphine equivalents (OMEs) during a 1-year period. $^{3}$ It also must be noted that more than $18 \%$ of patients in that study did not use any opioids in the week after surgery.

In another study, Overton et al. ${ }^{4}$ convened a multidisciplinary panel that included patients to determine best practices for postoperative opioid prescribing. Their discussion was informed by a literature search, and prescribing recommendations were based on experience. They found that the patient participants who had undergone the procedures consistently recommended smaller opioid prescriptions than the surgeons who performed the procedures. Thus, the presumption by Woeste and colleagues that patients filled and took opioids as prescribed likely overestimated actual patient use significantly.

Currently, recommendations for opioid prescribing for patients undergoing mastectomy are available. ${ }^{5,6}$ Scully et al. $^{5}$ have recommended opioid prescriptions for a duration 4-13 postoperative days. Another frequently used resource, the Opioid Prescribing Engagement Network (OPEN) from the Michigan Surgical Quality Collaborative, recommends that patients undergoing mastectomy with or without sentinel lymph node biopsy be administered up to 20 5-OME-strength tablets and those undergoing modified radical mastectomy be administered up to 30 5-OME-strength tablets. ${ }^{6}$ These 
recommendations do not account for patient or surgical factors that may increase postoperative opioid needs. The recommendations were published about the time of the data collection for this study and thus were likely not used for the study patients.

It is critical that all providers who prescribe postoperative opioids be aware of these recommendations and use them regularly. It is not surprising that the authors of this study found that patients with prescriptions for more opioids used more opioids. This finding, consistent with other recently published data, strengthens the argument that it is critical for providers to prescribe only appropriate amounts of postoperative opioids. ${ }^{7}$ It also is important for providers to realize that prescribing no opioids is acceptable and that patients who express a desire to avoid opioids should not be given an opioid prescription.,

Recently, Enhanced Recovery After Surgery (ERAS) programs have become more prevalent, with the goals of standardizing preoperative education and using optimal multimodality pain control strategies such that patients have an early return to preoperative functioning while also decreasing costs. ${ }^{8}$ It is important to recognize that data regarding the use of non-narcotic medications, including the use of a local anesthetic, were not available in this study. We know that the use of non-narcotic pain medications, including acetaminophen and ibuprofen, is critical for optimal postoperative pain control. ${ }^{9,10}$ As such, the education of patients in the preoperative setting is paramount to ensure appropriate expectations for postoperative pain control. This education should focus on using nonnarcotic medications, including acetaminophen and ibuprofen, as a first line for postoperative pain, with opioids used for breakthrough pain only. It should be emphasized to patients that use of non-narcotic medications may be sufficient for pain control in the postoperative period.

This study used the KASPER system to identify patients' last active opioid prescription. This strategy avoided the potential for recall bias, which is a limitation of other similar studies. As these electronic databases become available across the country, it is important that those who provide opioid prescriptions reference these databases such that patients using opioids in the preoperative setting may be identified. This recognition provides an important opportunity for interventions because these patients are known to be at increased risk for POU. ${ }^{1,7}$
Finally, this study used POU as a surrogate for pain control but did not evaluate pain control for patients undergoing mastectomy. As we seek to decrease opioid prescribing by using non-opioid strategies, we must be cognizant that we are maintaining adequate postoperative pain control for our patients. This has implication not only for postoperative recovery, but also for patient satisfaction scores, which have become increasingly important because of efforts to make these scores available to the public. As breast surgeons strive to reduce opioid prescribing while optimizing postoperative pain control, studies such as this provide important information such that prescriptions can be tailored to patient needs because one size does not fit all.

\section{REFERENCES}

1. Woeste MR, Bhutiani N, Geller AE, et al. Identifying factors predicting prolonged opioid use after mastectomy. Ann Surg Oncol. 2020. https://doi.org/10.1245/s10434-019-08171-4.

2. Lee JS, Hu HM, Edelman AL, et al. New persistent opioid use among patients with cancer after curative-intent surgery. $J$ Clin Oncol. 2017;35:4042-9.

3. Park KU, Kyrish K, Terrell J, et al. Surgeon perception versus reality: opioid use after breast cancer surgery. J Surg Oncol. 2019;119; 909-15.

4. Overton HN, Hanna MN, Bruhn WE, et al. Opioid-prescribing guidelines for common surgical procedures: an expert panel consensus. J Am Coll Surg. 2018;227:411-8.

5. Scully RE, Schoenfeld AJ, Jiang W, et al. Defining optimal length of opioid pain medication prescription after common surgical procedures. JAMA Surg. 2018;153:37-43.

6. Opioid Prescribing Engagement Network (OPEN) Prescribing Recommendations. Retrieved 20 December 2019 at https://mich igan-open.org/prescribing-recommendations/.

7. Park KU, Kyrish K, Yi M, et al. Opioid use after breast-conserving surgery: prospective evaluation of risk factors for high opioid use. Ann Surg Oncol. 2019. https://doi.org/10.1245/s104 34-019-08091-3.

8. Temple-Oberle C, Shea-Budgell MA, Tan M, et al. Consensus review of optimal perioperative care in breast reconstruction: Enhanced Recovery After Surgery (ERAS) Society recommendations. Plast Reconstr Surg. 2017;139:1056e-1071e.

9. Lee JS, Howard RA, Klueh MP, et al. The impact of education and prescribing guidelines on opioid prescribing for breast and melanoma patients. Ann Surg Oncol. 2019;26:17-24.

10. Rojas KE, Manasseh DM, Flom PL, et al. A pilot study of breast surgery Enhanced Recovery After Surgery (ERAS) protocol to eliminate narcotic prescription at discharge. Breast Cancer Res Treat. 2018;171:621-6.

Publisher's Note Springer Nature remains neutral with regard to jurisdictional claims in published maps and institutional affiliations. 